\title{
INFECTIONS AND ANTIMICROBIAL USE AMONG INSTITUTIONALIZED RESIDENTS IN HUNGARY: INCREASING NEED OF MICROBIOLOGICAL SURVEILLANCE
}

\author{
RITA SZABÓ* and KAROLINA BÖRÖCZ \\ National Centre for Epidemiology, Department of Hospital Epidemiology and Hygiene, \\ Budapest, Hungary
}

(Received: 27 August 2014; accepted: 30 October 2014)

\begin{abstract}
As a result of the age-related changes, more elders live in long-term care facilities (LTCFs). Due to their susceptibility, infections and excess use of antimicrobials are common. The aim was to estimate the burden of infections and antimicrobial use in Hungarian LTCFs in order to increase the attention given to the prevention. European-wide point prevalence survey was conducted between April and May 2013. For each resident who had a signs and symptoms of an infection and/or treated with an antibacterial for systemic use a resident questionnaire was completed. Descriptive statistics were used to present the data. In total, 91 LTCFs with 11,823 residents were selected in this survey. The 252 residents had a sign/symptom of an infection $(2.1 \%)$ and 156 received antimicrobial (1.3\%). Skin and soft tissues $(36.5 \%)$ was the most frequent infection. However, antimicrobials were mostly prescribed for respiratory tract infections $(40.4 \%)$. The most common therapeutic antimicrobial agent (97.5\%) belonged to the quinolone antibacterials (34.2\%). Our results emphasise the need for targeted improvement of antimicrobial use including: reducing the use of quinolone antibacterials in order to prevent the spread of Clostridium difficile and other antimicrobial resistant microorganisms among institutionalized residents.
\end{abstract} Hungary

Keywords: elderly, long-term care facilities, infections, antimicrobial use,

\section{Introduction}

The number of people aged 65 years and over is increasing worldwide. In Hungary, the number of elderly people has increased from 1,372,661 in 1960 to more than $2,364,420$ to date and is growth with $0.2 \%$ annually [1]. As a result

* Corresponding author; E-mail: szabo.rita@oek.antsz.hu 
of the age-related changes (e.g. impaired mobility, incontinence and disorientation) and changed healthcare system (e.g. early hospital discharge), more and more elders (39,847 in 2000 and 51,736 in 2012) live in long-term care facilities (LTCFs), corresponding to approximately $5 \%$ of the total Hungarian elderly population [1].

Residents of LTCFs are at high risk at infections due to their increased susceptibility (e.g. immunosenesence) and institutional factors (e.g. low priority of infection prevention and control measures) [2]. Recent reports with different methodologies indicated that the prevalence of infections is between $2.6 \%$ and $11.3 \%$ [3-4]. Because of infections, residents are often treated with antimicrobial agents. The most important adverse outcome of the excess use of antimicrobial agents is the emergence of multidrug resistant microorganisms, such as methicillin-resistant Staphylococcus aureus (MRSA), vancomycin-resistant Enterococcus spp. (VRE) [5-9].

Due to the lack of EU-wide cross-country comparable data on infections and antimicrobial use in LTCFs, the European Centre for Disease Prevention and Control (ECDC) funded the HALT project (Healthcare Associated Infections in Long-Term Care Facilities) in 2008. The aims were to estimate the burden of infections and antibiotic use and to collect data on infection control practices in order to increase the attention given to the prevention in LTCFs.

After a pilot survey in 2009 (including 4 Hungarian LTCFs), a first EUwide point prevalence survey was performed between May and September 2010, including 25 countries with 722 LTCFs (including 42 Hungarian LTCFs). Based on the experience of the first HALT, the methodology was slightly improved and a repeated survey (HALT-2) was set up in 2013 including 19 countries with 1,181 LTCFs [10-11].

This article presents the Hungarian results from the HALT-2 project, focusing on the burden of infections and antimicrobial use in participating LTCFs.

\section{Methods}

The European-wide repeated point-prevalence survey was conducted between April and May 2013 in 19 countries. Based on the protocol, the national coordinating institute (National Center for Epidemiology, NCE) organised the survey during the suggested period [11]. All LTCFs (e.g. general nursing care, residential care, psychiatric care, mixed care) with 50 beds and over (in total, 420 LTCFs; $24 \%$ of all Hungarian LTCFs) were invited to participate voluntary in 
this survey. Furthermore, it was recommended that the residents in these LTCFs need constant supervision and high-skilled nursing care, but do not need invasive medical procedures (e.g. mechanical ventilation). Hospital long-term care wards, residential care (e.g. hotel without any kind of nursing care), sheltered care houses, day centers, home-based centers, resident flat and protected living were excluded.

Within the participating LTCF, a resident was considered eligible for the survey if 1) she/he lived permanently in the LTCF, 2) had resided there for at least one day and 3) was present at $8 \mathrm{AM}$ on the day of the survey. All eligible residents had to be included. Residents hospitalized on the survey day were excluded.

According to the protocol, data were collected at LTCF and resident level (for the latter, including infection and antimicrobial use data if any) on standardised data collection forms (paper-based questionnaires). The LTCF questionnaire collected data on structural and functional characteristics of LTCF demographics (e.g. availability of qualified nursing $24 / 24 \mathrm{~h}$, total number of resident rooms), denominator data (e.g. predisposing factors of all eligible residents) and information on infection control practices and antibiotic policy in the LTCF (e.g. number of infection control staff, presence of written protocols for prevention, total annual consumption of alcohol hand rubs, use of restrictive list of antimicrobial agents).

For each eligible resident who showed a sign/symptom of an active infection and/or treated with an antibacterial agent on the day of the survey, a resident questionnaire was completed. This form also included questions on resident demographics (e.g. age, sex, length of LTCF stay, admission to an acute care hospital in the last three months, surgery in the previous 30 days) and predisposing factors (e.g. presence of urinary catheter and/or vascular catheter, pressure sore or other wounds, urinary and/or faecal incontinence, disorientation in time and/or space, mobility). Infection data included the infection type corresponding to one of the case definitions by applying decision algorithm. Data on antimicrobial use included the antimicrobial name, administration route, type of treatment (prophylactic or therapeutic), indication for antimicrobial use (the site of diagnosis for treatment intention of an infection), place and person of prescription, isolated microorganisms and selected antimicrobial resistance data (oxacillin-sensitive/resistant Staphylococcus aureus, glycopeptides-sensitive/resistant Enterococcus species, $3^{\text {rd }}$ generation cephalosporin and carbapenem-sensitive/resistant Enterobacteriaceae, carbapenem-sensitive/resistant Pseudomonas aeruginosa, carbapenem-sensitive/resistant Acinetobacter baumannii). 


\section{Case definitions for infections}

The diagnosis of an infection in elderly residents can be difficult due to the atypical presentation of infections (e.g. pyrexia is not sensitive indicator of infection), decline in mental and/or cognitive functions (e.g. disorientation). In addition, diagnostic testing (e.g. radiology, microbiologic sampling) for detection and confirmation of an infection is infrequent in LTCFs. Thus Centers for Disease Control and Prevention (CDC) case definitions for infections were used where these had been developed previously for LTCFs by McGeer et al. [12]. For the purposes of the protocol, an infection was defined as active on the day of the survey when signs and symptoms of the infection were present on the survey day or presented in the past and the resident were (still) received antimicrobial treatment for that infection on the survey day.

\section{Inclusion of antimicrobial agents}

For antimicrobial use, the Anatomical Therapeutic Chemical (ATC) classification system of the World Health Organization (WHO) Collaborating Centre for Drug Statistics Methodology was used [13]. All oral, rectal, intramuscular and intravenous treatment with antibacterials, antimycotics and antimycobacterials for systemic use and antibiotic treatment by inhalation were included. Antivirals for systemic use, antimicrobials for topical use and antiseptics were not included.

\section{Data collection and process}

Participating LTCFs were asked to choose one single day in the course of the survey to collect all necessary data prospectively. The protocol recommended that data from any single LTCF should all be collected on a single day. The total time frame for data collection for all wards of a large single LTCF was recommended not to exceed two consecutive days during the survey period.

\section{Training}

Because of it was important that surveyors record all signs and symptoms of infections on resident questionnaire to allow infection prevalence to be precisely estimated, training of LTCF staff was considered a priority throughout the preparation of the survey. In March 2013, six, one-day national training 
courses were given by the NCE to explain the methodology and to instruct the data collection to the LTCF staff. Standardised training curriculum and material was provided in English by the ECDC.

Data delivery

Collected data on paper forms were sent to the NCE by each LTCF and after data cleaning, these data were inputted into a stand-alone software programme. An electronic copy of data from each LTCF was emailed securely to the ECDC EU-wide database without resident-identifying data.

\section{Data analysis}

We used descriptive statistics to summarise resident data - number (\%) for categorical variables and median (interquartile range, IQR) for continuous variables. 95\% confidence intervals (CI) were calculated for the infection rate using with the Poisson distribution. The Mann-Whitney $U$-test was used to compare the differences between the prevalence with the infections, the three most frequent types of infections and the antimicrobial use stratified by type of demographics and predisposing factors. A $P$ value of less than 0.05 was considered statistically significant for all analysis. Statistical analyses were performed using EpiData version 3.1 (http://www.epidata.dk).

The prevalence of infections was reported as the percentage of residents with at least one infection over the total number of eligible residents. For infection types and isolated microorganism, relative frequencies were reported using the total number of infections or isolated microorganisms as the denominator.

The prevalence of antimicrobial use was reported as the percentage of residents receiving at least one antimicrobial agent over the total number of eligible residents. For antimicrobials, relative frequencies among the total number of antimicrobials are given as the denominator.

\section{Ethical consideration}

Because of the survey did not entail any direct contact with patients, ethical approval was not important. Confidentiality of data was assured by the use of a unique study number for each participating LTCFs and eligible residents allotted by the NCE and/or by the surveyors. 


\section{Results}

A total of 91 LTCFs with 11,823 eligible residents were selected in this survey. The median size of the participating LTCFs was 102.5 beds and varied from 26 beds to 690 beds. Only 19.8\% of LTCFs had single rooms for isolation.

The majority of all eligible residents were female (63.6\%). The median age in the residents surveyed was 69 years (IQR, 58-97 years). More than $20.8 \%$ of eligible residents were older than 85 years. The most common predisposing factors were the faecal and/or urinary incontinence (43.9\%), followed by impaired mobility (i.e., wheelchair bound or bedridden) (36.5\%) and disorientation in time and/or space $(29.9 \%)$. The occurrence of urinary catheter $(1.4 \%)$, recent surgery $(0.6 \%)$ and vascular catheter $(0.1 \%)$ was very limited.

\section{Infections}

On the day of the survey, 252 residents were presented signs/symptoms of an infection. The prevalence of infections was 2.1\% (IQR, 0-10.1\%). Of all infection groups, infections of skin and soft tissues $(36.5 \%)$ were the most frequently presented on the day of the survey, followed by the infections of respiratory tract $(28.6 \%)$ and urinary tract $(21.8 \%)$. The group of skin and soft tissue infections primarily comprised of cellulitis, soft tissue or wound infections $(80.4 \%)$. Scabies (13\%), fungal infection (5.4\%) and herpes simplex or zoster infection $(1.1 \%)$ were not common in these settings. The respiratory infection group mostly included common cold or pharyngitis (51.4\%) and other lower respiratory tract infection (31.9\%). Among urinary tract infections, non-indwelling-catheterrelated urinary tract infections were the most frequent (98.2\%). Detailed information of the distribution of infections among the eligible residents can be found in Table I.

\section{Antimicrobial use}

156 residents were received at least one antimicrobial agents. The prevalence of antimicrobial use was $1.3 \%$ (IQR, $0-6.4 \%$ ). $96.8 \%$ of reported antimicrobials were given for treatment of an infection. The most common indication for therapeutic antimicrobial use was the treatment of respiratory tract infections $(40.9 \%)$, urinary tract infections $(36.4 \%)$ and skin and soft tissue infections $(15.9 \%)$ (Table I). The most common therapeutic antimicrobial agent $(97.5 \%)$ belonged to the ATC J01 class of 'antibacterials for systemic use'. The most impor- 


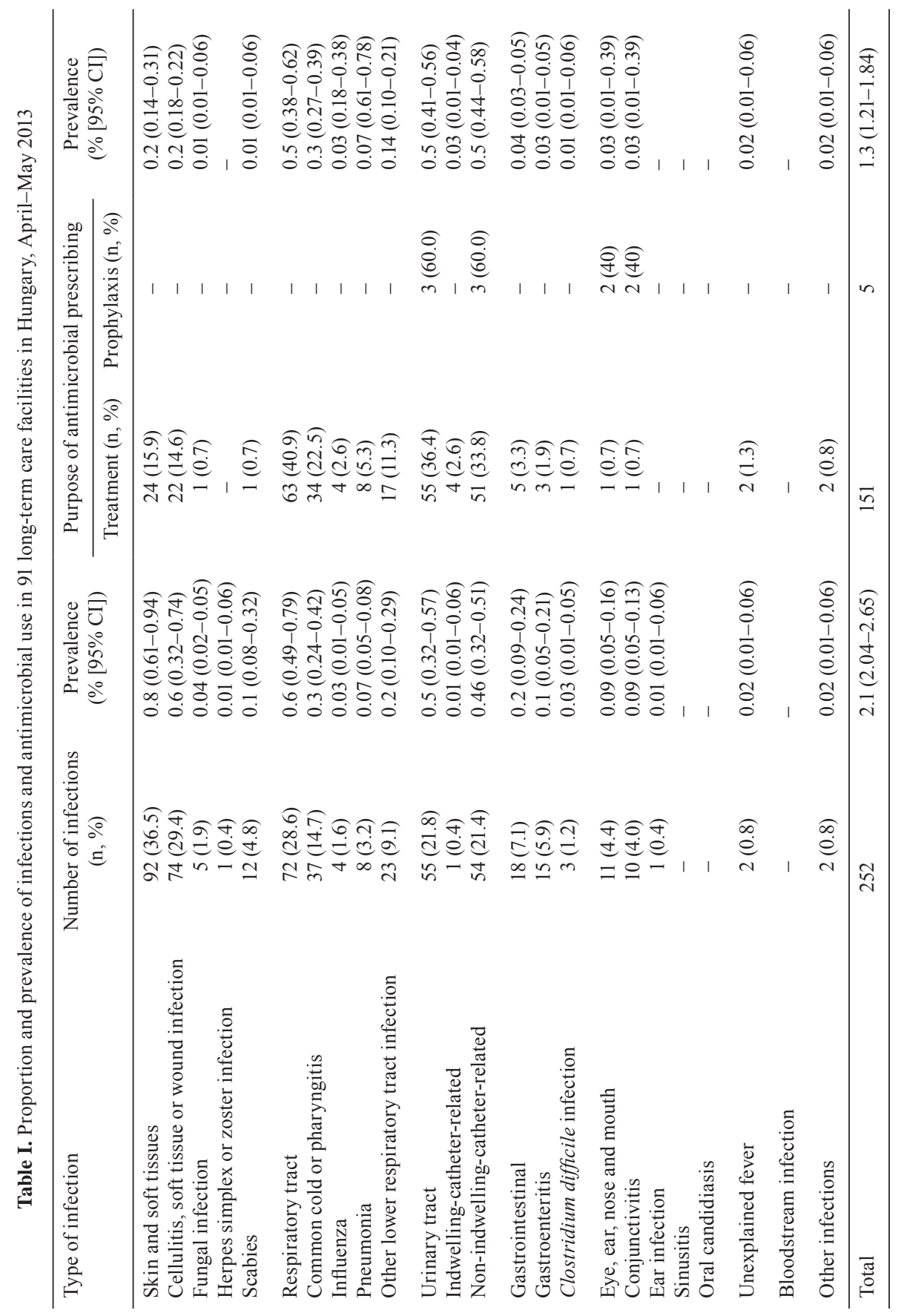




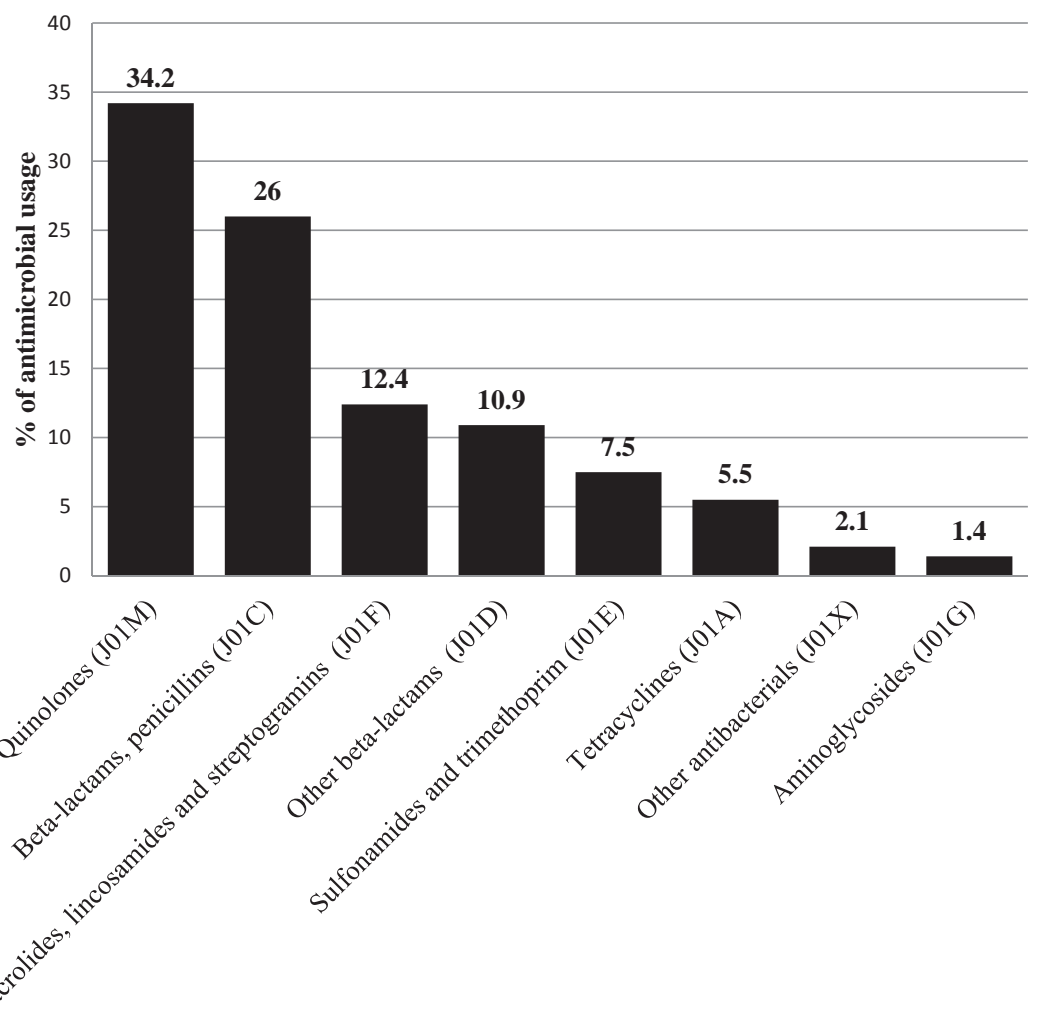

Figure 1. Distribution of use of J01 antibacterials for systematic use in 91 long-term care facilities in Hungary, April-May 2013

tant J01 subclasses were J01M quinolone antibacterials (34.2\%), J01C beta-lactam antibacterials (26\%), J01F macrolides, lincosamides and streptogramins (12.4\%), and J01D other beta-lactam antibacterials (10.9\%) (Fig. 1).

The most commonly prescribed empirically treatments for respiratory tract infections were beta-lactam antibacterials $(40.9 \%)$, followed by other betalactam antibacterials (21.3\%) and macrolides, lincosamides and streptogramins (14.8\%). The most often empirically prescribed antibacterials for urinary tract infections were quinolone antibacterials (64\%), followed by sulfonamides and trimethoprim (15.6\%) and beta-lactam antibacterials (7.8\%). The most frequent antibacterials prescribed for therapy of skin and soft tissue infections were betalactam antibacterials (47.4\%), followed by macrolides, lincosamides and streptogramins $(26.3 \%)$ and tetracyclines $(10.5 \%)$.

Only $2.4 \%$ of antimicrobials were microbiologically documented treatments. Three positive microbiology results were available for gastrointestinal 
infection (Clostridium difficile), two for urinary tract infection (Enterobacter cloacae, Escherichia coli) and one for other infection (Staphylococcus aureus). No resistant strains were found.

A total of $3.2 \%$ of all prescribed antimicrobial agents was used for prophylactic purposes. Antimicrobials were prescribed for the prevention of urinary tract infections (60\%) and ear, nose, mouth infections (40\%) (Table I).

$84.4 \%$ of participating LTCFs had no antimicrobial stewardship elements. The most frequent components of antimicrobial policies in the LTCFs were 'restrictive list of antimicrobial to be prescribed' (18.9\%), 'use of written guidelines' (7.8\%) and 'feedback to physicians on institutional antimicrobial consumption' (4.4\%). In LTCFs which had a restrictive list of antimicrobials to be prescribed, $73.7 \%$ of these settings restricted only intravenously administered antimicrobials.

\section{Predisposing factors}

Among eligible residents with at least one infection and antimicrobial agent, $65.1 \%$ and $61.6 \%$ were female. The median age was 78 and 77 years. The most frequent predisposing factors were impaired mobility $(68.5 \%$ and $63.5 \%)$, followed by incontinence $(63.9 \%$ and $61.6 \%)$ and disorientation $(42.4 \%$ and $35.8 \%$ ). The association of infections and antimicrobial use with demographics and predisposing factors can be found in Table II.

\section{Discussion}

In the last decade, several European countries set up initiatives to increase awareness for the problem of infections and antimicrobial use in their LTCFs. The aim of the HALT-2 project in the EU Member States was to collect aggregated data on infections and use of antimicrobial agents among elderly residents in LTCFs. At the national level, this survey gave insight into the burden of infections and antimicrobial use in Hungarian LTCFs.

In Hungary 91 LTCFs participated in this survey. Residents of participating LTCFs were elderly (more than one-forth aged over 85 years) and presented a heavy care load (more than one-third of the eligible residents surveyed incontinent, immobile or disoriented) for the staff. This results are similar to the findings from other participating European LTCFs where the mean of residents with over 85 years, incontinence, disorientation and impaired mobility $(46.5 \%, 65.8 \%$, $54.9 \%$ and $52.6 \%$ ) were also high [11]. 


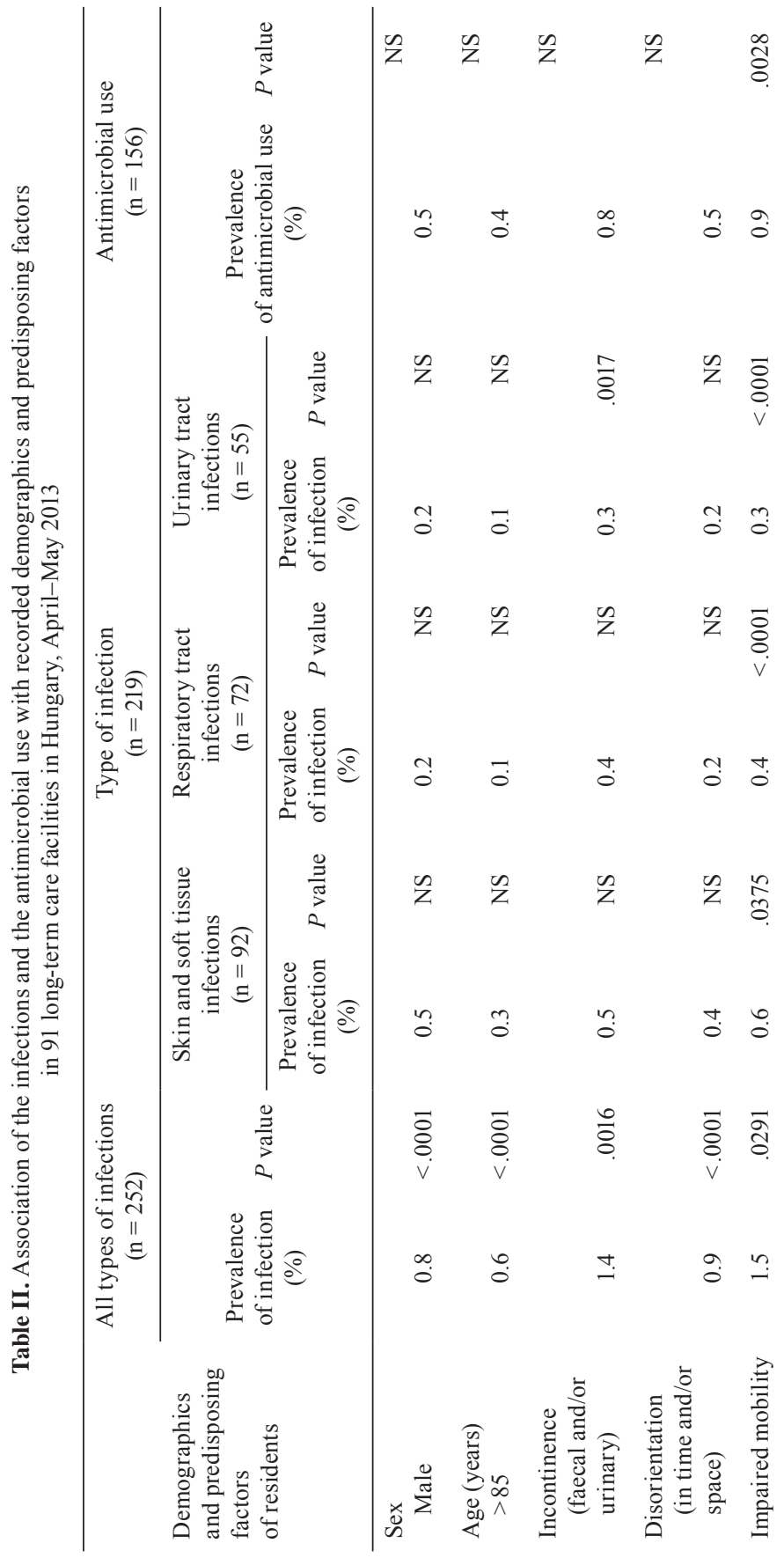




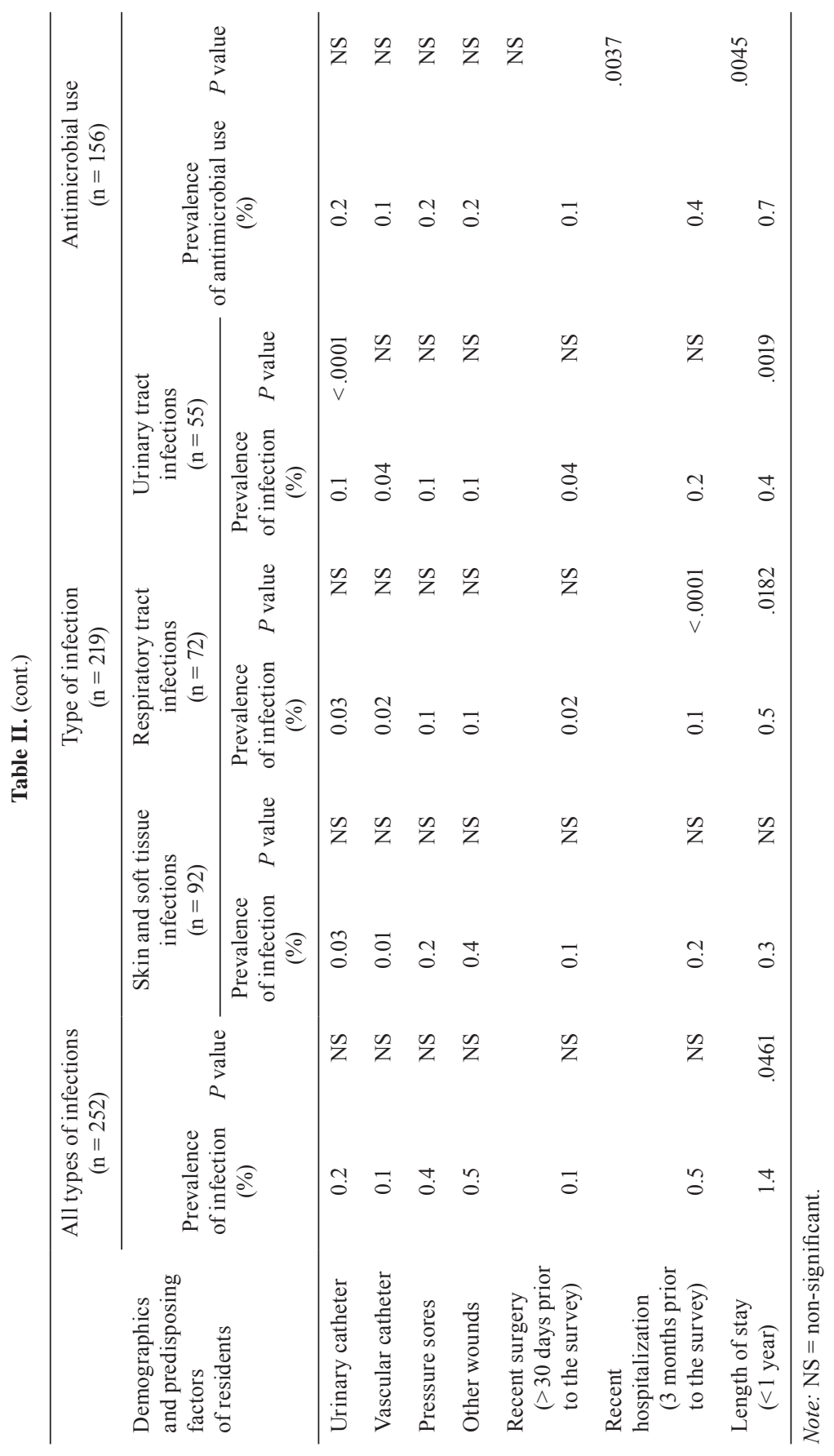


The collected data allowed for the estimation of the prevalence of infections and antimicrobial use. The prevalence of infection among residents in Hungarian participating LTCFs was $2.1 \%$. The infection prevalence rate was lower than those found in other European countries: $2.8 \%$ and $7.3 \%$ in Dutch LTCFs, $4.3 \%$ in German LTCFs, $6.4 \%$ in Irish LTCFs and $7.6 \%$ in Norwegian LTCFs $[11,14-18]$. Lower infection prevalence results were probably due to the general diagnostic problems (e.g., change in mental and/or cognitive status of elders), the lack of microbiological and radiological diagnostic testing, and/or good quality of LTCF nursing care. In addition, this finding suggested the negative influence of reporting behaviour, despite the similar methodology, and the positive influence of qualified nursing staff, because they can detect the signs and symptoms of an infection correctly. In our survey, skin and soft tissues infections $(36.5 \%)$ was the most frequent, followed by the infection of respiratory tract $(28.6 \%)$ and urinary tract $(21.8 \%)$. However, in contrast with other recent studies that found urinary tract infections to be the most frequent infection, following by respiratory tract infections and skin and soft tissues infections [11, 15, 19-20]. The lower frequency of urinary tract infections can be explained by the infrequent use of urinary catheter in Hungarian LTCFs.

The prevalence of antimicrobial use among participating LTCFs was lower (1.3\%) compared with results from other European surveys (2.4-15\%) [3, 11, 21-23]. National result showed that the prevalence of antimicrobial use was lower than the prevalence of infections, thus the overuse of antimicrobials is not common in participating LTCFs. Although prescription of quinolone antibacterials was frequent (34.2\%) in LTCFs, which can lead the increased presence of infections caused by Clostridium difficile and other antimicrobial resistant pathogens [22-27]. This result is similar to several overseas studies that quinolones are the most frequently prescribed antibacterials in LTCFs [20-27]. Among eligible residents receiving antibacterial agent for treatment of an infection, only $2.4 \%$ of prescriptions were based microbiologically results. In comparison with the results from Norwegian and Dutch surveys (5.8\% and 6.6\%), our culture sampling rate was low, probably due to the fact that microbiological diagnostic tests were not commonly requested by the physicians in the elderly care in Hungary $[14,18]$. Antibiotic stewardship resources were uncommon in these settings. Local data on antimicrobial resistance profiles, antimicrobial committee, feedback to the physicians on institutional antimicrobial consumption, advice from a pharmacist were available in less than $16 \%$ of the participating LTCFs. Similar to other European LTCFs, regular education sessions of prescribers is an important and urgent action for improvement in Hungarian LTCFs [11, 28].

Our survey had some limitations. Firstly, the representativeness of the survey sample was poor, although there was not a required sample size. Repre- 
sentativeness was less than optimal because LTCFs participated on a voluntary basis (convenient sample). Thus, our results could be heavily biased as a result of the very low number of participating LTCFs. Secondary, our national prevalence rates result from aggregating prevalence in individual LTCFs; therefore, these data must be interpreted with caution. Thirdly, low sensitivity (false negative or underreporting) of infections could be a problem, which may be related to the difficulty in confirming the case definition of an infection if sign and symptoms were not well documented in the residents' medical record or diagnoses of infection made were not always correct or because of this survey relied on self-administered data. Finally, we were not able to analyse the epidemiology of microorganisms, owing to the small number of microbiological samples performed. Several of these limitations may be improved in future surveys by increasing the number of participating LTCFs, enhanced training of LTCF staff in case definitions or reinforcing efforts to improve the quality of residents' medical records.

Despite these limitations, the HALT-2 project was the largest point-prevalence survey performed to date which was a great opportunity to get a comprehensive epidemiological overview of infections and use of antimicrobial agents in Hungarian LTCFs.

Based on our results, while a first important step has been made improving skills in surveillance of infections and antimicrobial use and raising awareness in Hungarian LTCFs, extensive training on infection prevention and control to healthcare practitioners is needed. In addition, there is a need for 1) implementing national guidelines (e.g., hand hygiene, antimicrobial stewardship); 2) developing infection prevention and control programme including recommendations on organisational and structural arrangements, therapeutic procedures, resource requirements, surveillance objectives and training; and 3) establishing infection surveillance system specific for LTCFs in order to prevent unnecessary suffering of elderly people. In addition, this survey identified an important area for targeted improvement of antimicrobial use including: reducing the use of quinolone antibacterials in order to prevent the spread of Clostridium difficile and other antimicrobial resistant microorganisms among residents of LTCFs. Complex (i.e., dialectic and interactive) trainings, providing feedback to physicians about their prescribing patterns, and locally developed antimicrobial stewardship may improve the quality of antibiotic prescribing [29-31]. For additional research, we consider it necessary to also take in consideration other factors (e.g., determination of risk factors for preventable infections, attributable morbidity and mortality) in order to explore which efforts help to prevent infections. Furthermore, other detailed information on the cost of infections is needed to assist efforts at prioritizing the national health policies. 


\section{Acknowledgements}

The authors thank all participating long-term care facilities and their surveyors who attended the training sessions and completed the questionnaires. We also thank our colleagues (Andrea Kurcz MD, Ágnes Hajdu MD, Ida Prantner MD, Andrásné Szőnyi, István Veress and Emília Süveges) for their professional support. Without whom this survey would not have been possible.

\section{Funding}

The HALT project was funded and supported by the European Centre for Disease Prevention and Control (ECDC): ECDC/2011/023.

\section{Disclaimer}

The information contained in this manuscript does not necessarily reflect the opinion of the European Centre for Disease Prevention and Control (ECDC).

\section{References}

1. Hungarian Central Statistical Office: Demographic Yearbook. Hungarian Central Statistical Office, Budapest, 2011, pp. 22-26.

2. Gavazzi, G., Krause, K.H.: Ageing and infection. Lancet Infect Dis 11, 659-666 (2002).

3. Moro, M.L., Mongardi, M., Marchi, M., Taroni, F.: Prevalence of long-term care acquired infections in nursing and residential homes in the Emilia-Romagne region. Infection 35, 250-255 (2007).

4. Nicolle, L.E.: Infection control in long-term care facilities. Clin Infect Dis 31, 752-756 (2000).

5. van Buul, L.W., van der Steen, J.T., Veenhuizen, R.B., Achterberg, W.P., Schellevis, F.G., Essink, R.T.G.M., van Benthem, B.H.B., Natsch, S., Hertogh, C.M.P.M.: Antibiotic use and resistance in long term care facilities. J Am Med Dir Assoc 13, 568.el-13 (2012).

6. Elizaga, M.L., Weinstein, R.A., Hayden, M.K.: Patients in long-term care facilities: A reservoir for vancomycin-resistant Enterococci. Clin Infect Dis 34, 441-446 (2002).

7. Bergman, P., Hogan, D.B., Fox, R.A.: The atypical presentation of infection in old age. Age Ageing 16, 201-207 (1987).

8. Mehr, D.R., Binder, E.F., Kruse, R.L., Zweig, S.C., Madsen, R.W., D’Agostino, R.B.: Clinical findings associated with radiographic pneumonia in nursing home residents. J Fam Pract 50, 931-937 (2001). 
9. Metlay, J.P., Kapoor, W.N., Fine, M.J.: Does this patient have community-acquired pneumonia? Diagnosing pneumonia by history and physical examination. J Am Dir Med Assoc 278, 1440-1445 (1997).

10. European Centre for Disease Prevention and Control (ECDC): Point prevalence survey of healthcare-associated infections and antimicrobial use in European long-term care facilities, May-September 2010. Stockholm: ECDC; 2014. Available from: http://www.ecdc. europa.eu/en/publications/Publications/healthcare-associated-infections-antimicrobialconsumption-point-prevalence-survey-long-term-care-facilities-2010.pdf

11. European Centre for Disease Prevention and Control (ECDC): Point prevalence surveys of healthcare-associated infections and antimicrobial use in European long-term care facilities, April-May 2013. Stockholm: ECDC; 2014. Available from: http://www.ecdc. europa.eu/en/publications/Publications/healthcare-associated-infections-point-prevalencesurvey-long-term-care-facilities-2013.pdf

12. McGeer, A., Campbell, B., Emori, T.G., Hierholzer, W.J., Jackson, M.M., Nicolle, L.E.: Definitions of infection for surveillance in long-term care facilities. Am J Infect Control 19, 1-7 (1991).

13. World Health Organization (WHO): The Anatomical Therapeutic Chemical Classification System with Defined Daily Doses (ATC/DDD). Geneva: WHO; 2013. Available from: http://www.who.int/classifications/atcddd/en/

14. Eikelenboom-Boskamp, A., Cox-Claessens, J.H.M., Boom-Poels, P.G.M., Drabbe, M.I.J., Koopmans, R.T.C.M., Voss, A.: Three-year prevalence of healthcare-associated infections in Dutch nursing homes. J Hosp Infect 78, 59-62 (2011).

15. Cotter, M., Donlon, S., Roche, F., Byrne, H., Fitzpatrick, F.: Healthcare-associated infection in Irish long-term care facilities: results from the First National Prevalence Study. J Hosp Infect 80, 212-216 (2012).

16. Eilers, R., Veldman-Ariesen, M.J., Haenen, A., van Benthem, B.H.: Prevalence and determinants associated with healthcare-associated infections in long-term care facilities (HALT) in the Netherlands, May to June, 2010. Euro. Surveill 17, pii: 20252 (2012).

17. Heudorf, U., Boehlcke, K., Schade, M.: Healthcare-associated infections in long-term care facilities (HALT) in Frankfurt am Main, Germany, January to March 2011. Euro. Surveill 17, pii: 20256 (2012).

18. Eriksen, H.M., Iversen, B.G., Aavitsland, P.: Prevalence of nosocomial infections and use of antibiotics in long-term care facilities in Norway, 2002 and 2003. J Hosp Infect 57, 316-320 (2004).

19. Andersen, B.M., Rasch, M.: Hospital-acquired infections in Norwegian long-term care institutions. A three-year survey of hospital-acquired infections and antibiotic treatment in nursing/residential homes, including 4500 residents in Oslo. J Hosp Infect 46, 288-296 (2000).

20. Loeb, M.B., Craven, S., McGeer, A.J., Simor, A.E., Bradley, S.F., Low, D.E., ArmstrongEvans, M., Moss, L.A., Walter, S.D.: Risk factors for resistance to antimicrobial agents among nursing homes residents. Am J Epidemiol 157, 40-47 (2003).

21. Blix, H.S., Bergman, J., Schjott, J.: How are antibacterials used in nursing homes? Results from a point-prevalence prescription study in 44 Norwegian nursing homes. Pharmacoepidemiol Drug Saf 19, 1025-1030 (2010).

22. McClean, P., Hughes, C., Tunney, M., Goossens, H., Jans, B.: Antimicrobial prescribing in European nursing homes. J Antimicrob Chemother 66, 1609-1616 (2011). 
23. D'Agata, E., Mitchell, S.L.: Patterns of antimicrobial use among nursing home residents with advanced dementia. Arch Intern Med 168, 357-362 (2008).

24. Deshpande, A., Pasupuleti, V., Thota, P., Pant, C., Rolston, D.D., Sferra, T.J., Hernandez, A.V., Donskey, C.J.: Community-associated Clostridium difficile infection and antibiotics: a meta-analysis. J Antimicrob Chemother 68, 1951-1961 (2013).

25. Marwick, C.A., Yu, N., Lockhart, M.C., McGuigan, C.C., Wiuff, C., Davey, P.G., Donnan, P.T.: Community-associated Clostridium difficile infection among older people in Tayside, Scotland, is associated with antibiotic exposure and care home residence: cohort study with nested case-control. J Antimicrob Chemother 68, 2927-2933 (2013).

26. Mylotte, J.M., Keagle, J.: Benchmarks for antibiotic use and cost in long-term care. J Am Geriatr Soc 53, 1117-1122 (2005).

27. Pakyz, A.L., Dwyer, L.L.: Prevalence of antimicrobial use among United States nursing home residents: results from a national survey. Infect Control Hosp Epidemiol 31, 661662 (2010).

28. Latour, K., Catry, B., Broex, E., Vankerckhoven, V., Muller, A., Stroobants, R., Goossens, H., Jans, B.: Indications for antimicrobial prescribing in European nursing homes: results from a point prevalence survey. Pharmacoepidemiol Drug Saf 21, 937-944 (2012).

29. Fleming, A., Browne, J., Byrne, S.: The effect of interventions to reduce potentially inappropriate antibiotic prescribing in long-term care facilities: a systematic review of randomised controlled trials. Drugs Aging 30, 401-408 (2013).

30. McClean, P., Tunney, M., Gilpin, D., Parsons, C., Hughes, C.: Antimicrobial prescribing in residential homes. J Antimicrob Chemother 67, 1781-1790 (2012).

31. Schwartz, D.N., Adiad, H., DeMarais, P.L., Armeanu, E., Trick, W.E., Wang, Y., Weinstein, R.A.: An educational intervention to improve antimicrobial use in a hospital-based long-term care facility. J Am Geriatr Soc 55, 1236-1242 (2007). 